\title{
Arterial Oxygen Desaturation and Intestinal Absorption of Xylose
}

\section{J. S. MILLEDGE}

British Medical fournal, 1972, 3, 557-558

\section{Summary}

Small-bowel absorption was studied using the xylose absorption test in 16 patients with varying degrees of arterial oxygen desaturation due to either congenital heart disease or chronic lung disease. Xylose absorption was decreased in the cases with more severe desaturation. The correlation of xylose absorption with arterial saturation was significant. In nine cases hypoxia was relieved by either oxygen administration or surgery. Repeat testing showed an increase in xylose absorption in every case, the mean increase being $11.7 \%$, which was statistically significant.

\section{Introduction}

In a number of widely different clinical situations hypoxaemia is associated with weight loss or failure to gain weight. Patients with progressive emphysema at a certain point in the progress of their disease lose weight and become emaciated. Children with congenital heart disease are stunted and underweight. Climbers remaining at high altitude lose weight even when they take an adequate diet (Pugh, 1962). In none of these conditions has any primary intestinal disorder been shown.

This study was undertaken to assess the effect of arterial oxygen desaturation on intestinal absorption by using xylose as a test substance. Patients with chronic respiratory disease or congenital cyanotic heart disease were studied before and after correcting their desaturation.

\section{Patients and Methods}

Sixteen patients aged 6-37 years with varying degrees of cyanosis were studied. Three had chronic respiratory disease, two with emphysema and one with bronchiectasis, and all had mild cor pulmonale. Of 13 patients with heart disease 10 had tetralogy of Fallot, two had tricuspid atresia, and one had transposition of the great vessels.

None of the cardiac cases were in cardiac failure. Preoperative drugs were limited to ferrous sulphate and in two cases propranolol. Postoperative drugs included digitalis, diuretics, and broad-spectrum antibiotics. The respiratory cases were treated with broad-spectrum antibiotics, bronchodilators, and diuretics. Diuretics were not given on the morning of a test.

The xylose absorption test was carried out as follows. A dose of $5 \mathrm{~g}$ of $\mathrm{D}$-xylose was taken in a glass of water at 5 a.m. No food was allowed for two hours. A normal breakfast was then given. All the urine passed until 10 a.m. was collected and measured, and an aliquot was analysed for xylose by the method of Roe and Rice (1948). The quantity of xylose passed was calculated and expressed as a percentage of the amount ingested. The test was carried out on two successive days and the results were averaged. The mean difference between duplicate tests was $3.2 \%$, maximum $8.6 \%$.

Christian Medical College and Hospital, Vellore, North Arcot District, S. India

J. S. MILLEDGE, M.D., F.R.C.P., Associate Professor, Department of Thoracic Surgery
Arterial blood was taken by femoral or brachial artery puncture and analysed for oxygen saturation in an American optical or Kipp hemoreflector, checked by frequent estimations using the Van Slyke apparatus. Arterial oxygen desaturation was relieved as follows. In the three patients with chronic lung disease oxygen was administered at 1 to $21 . / \mathrm{min}$, via a nasal catheter. This resulted in an increase in arterial oxygen saturation from $61-70 \%$ to $90-94 \%$. Oxygen was begun at 9 p.m. the previous evening and continued until the end of the xylose test at 10 a.m. the next day.

Of the six patients with cyanotic heart disease five had Fallot's tetralogy and underwent total correction of this defect, which resulted in an increase in arterial saturation from $56-83 \%$ to $94-98 \%$. The sixth patient with transposition of the great vessels had a Waterston's shunt with improvement of arterial saturation from $60 \%$ to $70 \%$.

\section{Results}

The results of initial xylose absorption tests are plotted against arterial oxygen saturation in Fig. 1. There was a definite correlation between percentage xylose absorption and

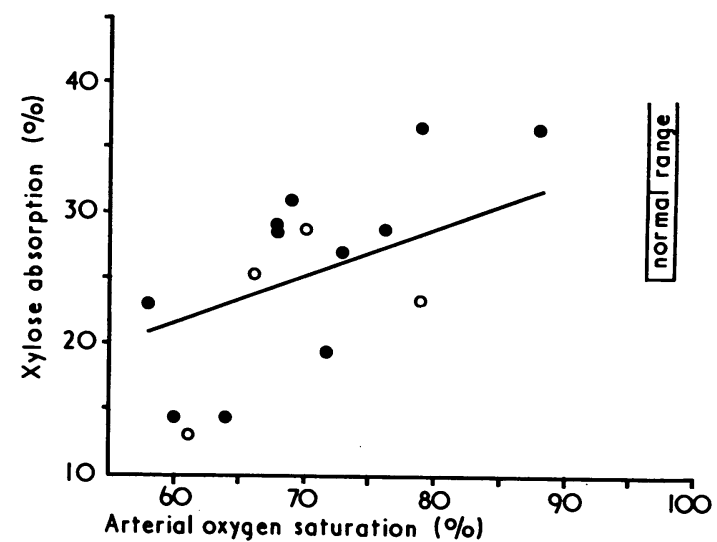

FIG. 1-Results of xylose absorption tests plotted against saturation in patients with either congenital cyanotic heart disease or chronic respiratory disease $O$. Linear regression line is shown. $r=0.72$ and is significant at the $1 \%$ level.

arterial oxygen saturation. The correlation coefficient was +0.72 , which is significant at the $1 \%$ level. In the three respiratory cases and six of the cardiac cases tests were repeated after relief of hypoxia. The results are shown in Fig. 2. There was an increase in xylose absorption in every case, the mean increase being $11.7 \%$. This change is statistically significant $(P<0.001$ on paired $t$ test $)$.

\section{Discussion}

The present findings suggest that hypoxia results in a reduction in absorption of xylose from the bowel. However, other possible explanations must be considered. A proportion of xylose absorbed is metabolized but it is usually assumed that the proportion remains constant so that the amount excreted is proportional to the absorption.

Could the present results be due to a change in the proportion of xylose metabolized? This explanation would necessi- 
tate an increase in the xylose metabolized in the hypoxic state compared with the normal state, and while this is possible it seems unlikely. There is also some tubular reabsorption of xylose by the kidney. A change in the proportion reabsorbed could account for these results if hypoxia increased the reabsorption of xylose; but again this seems unlikely. A large change in glomerular filtration rate might affect xylose excretion as it apparently does in aspirin

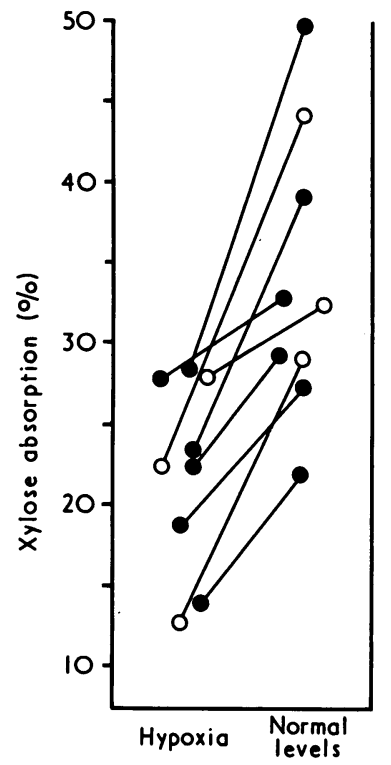

FIG. 2-Results of xylose absorption tests before and after relief of hypoxia. Each pair of symbols joined by a line represents one patient. $=$ Cyanotic heart disease. $O=$ Respiratory disease. Increase in absorption significan $(P=<0.001)$ on paired $t$ test.

ingestion (Kendall et al., 1971), but it has been shown that hypoxia of the degree found in these patients does not affect glomerular filtration rate (Berger et al., 1949).

Normal values for xylose absorption given in studies from the West are $35 \% \pm 6 \%$ and range from $23 \%$ to $48 \%$ (Sammons et al., 1967), and any value less than $25 \%$ is regarded as abnormal. But in India Baker et al. (1972) found that $24 \%$ of the apparently normal inhabitants of a South Indian village had values less than $25 \%$ and that $14 \%$ had less than $20 \%$ xylose absorption. In groups of patients with conditions where intestinal absorption is impaired, such as tropical sprue, values do tend to be lower on average and show improvement with relief of the condition. In the present study $6(37 \%)$ of the 16 patients had xylose absorption of less than $25 \%$ and $4(25 \%)$ less than $20 \%$. It was seen then that as a group these hypoxic patients did not have very much lower absorption values than the normal population. However, the less severely hypoxic patients in the group tended to have higher values and the more severely hypoxic lower values, resulting in a significant correlation between these two measurements.
Even more convincing evidence of the effect of hypoxia on xylose absorption was provided by the results before and after relief of hypoxia in the same patients, as shown in Fig. 2. Here, where each patient acts as his own control, the increase in absorption after relief of hypoxia is clearly seen.

In the initial results (Fig. 1) there was wide scatter. Firstly, the xylose absorption test showed considerable day-to-day variation in a single individual. Secondly, in the case of children with heart disease arterial saturation values obtained at the time of catheterization were used, which have been influenced by sedation or the disturbance of the procedure. Thirdly, the mucosal tissue $\mathrm{PO}_{2}$, which is presumably the crucial value for effective absorption, depends not only on arterial saturation but also on packed cell volume, local blood flow, and intercapillary distance-factors which must vary widely from patient to patient. Despite these considerations there was a significant correlation between arterial oxygen saturation and xylose absorption. This correlation and the significant increase in xylose absorption after relief of hypoxia are strongly suggestive of a causal relationship.

In the three respiratory cases oxygen administration for eight hours was sufficient to increase xylose absorption. This rather short time suggests the effect of hypoxia on the mucosa to be of a biochemical nature rather than a change in cell population.

A search of the literature did not uncover any similar study with which to compare these results, but a number of workers have measured xylose absorption in patients with anaemia due to various causes and after correction of anaemia (Varito, 1962; Guha and Rashmi, 1968; Mathan, 1970). In most patients they found rather low initial values which showed a rise after correction of the anaemia. As pointed out, the mucosal tissue $\mathrm{PO}_{2}$ is dependent on packed cell volume as well as arterial oxygen saturation, so the results support those of the present study, suggesting that a low tissue $\mathrm{PO}_{2}$ impairs absorption of xylose.

If this reduction in xylose absorption reflects a general intestinal malabsorption this could well account for the low weight of these and similar patients.

I am indebted to Dr. S. J. Baker and his associates for help and encouragement in this study, and to Mr. R. Jacob, who carried out the xylose estimations. I am also grateful to Drs. G. Cherian, I. P. Sukumar, and S. John for permission to study patients under their care.

\section{References}

Baker, S. J., Jacob, R., and Mathan, V. I. (1972). Indian fournal of Medical Research. 59, 1869 Berger, E. Y., Galaston, M., and Horowitz, S. A. (1949). Fournal of Clinical
Investigation, 28, 648.

Guha, D. K., and Rashmi, A. (1968). Indian fournal of Medical Research, 56, 1028 .

Kendall, M. J., Nutter, S., and Hawkins, C. F. (1971). British Medical fournal, 1, 533.

Mathan, V. I. (1970). Thesis for Ph.D., Madras University.

Pugh, L.G.C.E. (1962). British Medical fournal, 2, 621 .

Roe, J. H., and Rice, E. W. (1948). fournal of Biological Chemistry, 173, 507 Sammons, H. G., Morgan, D. B., Fraser, A. C., and Montgomery, R. D.
(1967). Gut, 8, 348. Varito, T. (1962). Scandinavian fournal of Clinical and Laboratory Investiga-
tion, 141, 36. 